\title{
TWO FOLIATIONS IN THE PLANE
}

\author{
JOHN M. FRANKS ${ }^{1}$
}

\begin{abstract}
This note gives two examples of foliations of an open set in the plane: a $C^{\infty}$ foliation with every leaf dense and a $C^{1}$ foliation with an exceptional minimal set.
\end{abstract}

In this note we give examples of two foliations of an open set in the plane. One is a $C^{\infty}$ foliation with every leaf dense and the other is a $C^{1}$ foliation with an exceptional minimal set. The region foliated in both cases is an open disk with three holes and the examples can be altered to give foliations on the plane minus three points.

In [1] Hector gives an example of a codimension one foliation of $R^{3}$ with every leaf dense and another with exceptional minimal sets. No such examples can exist for $R^{2}$ because such foliations would be orientable (i.e. the integral curves of a vector field) and the Poincaré-Bendixson theorem precludes the possibility of any minimal set for an orientable foliation in the plane except a single compact leaf. The examples constructed here are, of course, not orientable and hence not in conflict with the Poincaré-Bendixson theorem.

ADDED IN PROOF. It has been brought to the author's attention that the first of these examples was constructed some years ago by M. Dubois-Violette, but apparently never published.

These examples were inspired by a study of a very interesting diffeomorphism which has an invariant partial foliation-an example given by Plykin [3].

THEOREM. There exists a $C^{\infty}$ one dimensional foliation of an open region in $R^{2}$ with every leaf dense and a $C^{1}$ foliation of the same region with an exceptional minimal set.

Lemma. There exist diffeomorphisms $g_{1}, g_{2}, g_{3}$ and $g_{4}$ of the circle $S^{1}$ to itself such that:

(a) $g_{i}^{2}=\mathrm{id}$ for $i=1,2,3,4$; and each $g_{i}$ is orientation reversing with exactly two fixed points.

(b) $k_{1}=g_{1} \circ g_{2}$ is a $C^{\infty}$ diffeomorphism with every orbit dense.

(c) $k_{2}=g_{3} \circ g_{4}$ is a $C^{1}$ Denjoy diffeomorphism (i.e. has a Cantor set as minimal set).

Proof. Represent $S^{1}$ by the set of unit complex numbers $\left\{e^{i \theta}\right\}$ and define

Received by the editors February 6, 1975.

AMS (MOS) subject classifications (1970). Primary 57D30.

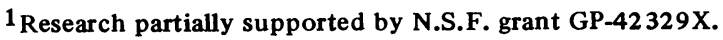

๑ American Mathematical Society 1976 
$g_{1}\left(e^{i \theta}\right)=e^{-i \theta}, g_{2}\left(e^{i \theta}\right)=e^{-i(\theta+r)}$ where $r / 2 \pi$ is irrational; then $k_{1}\left(e^{i \theta}\right)=$ $e^{i(\theta+r)}$ and $g_{1}^{2}=g_{2}^{2}=\mathrm{id}$.

Let $j: S^{1} \rightarrow S^{1}$ be defined by $j\left(e^{i \theta}\right)=e^{-i \theta}$. In order to define $g_{3}$ and $g_{4}$ it suffices to find a Denjoy diffeomorphism $k$ such that $j \circ k=k^{-1} \circ j$ because then $g_{3}=j$ and $g_{4}=j \circ k$ satisfy the conclusion $\left(g_{4}^{2}=j \circ k \circ j \circ k=\right.$ $j \circ k \circ k^{-1} \circ j=j^{2}=\mathrm{id}$ ). We refer now to the construction of Denjoy diffeomorphisms given in [2, pp. 47-49]. The idea is to take an irrational rotation $h$ : $S^{1} \rightarrow S^{1}\left(h\left(e^{i \theta}\right)=e^{i(\theta+r)}\right)$, insert an interval at each point of an orbit of $h$ and define the diffeomorphism on this enlarged circle. We will do the same except that it is necessary to insert two sequences of intervals.

Choose a point $x \in S^{1}$ such that the sets $\left\{h^{i}(x) \mid-\infty<i<\infty\right\}$ and $\left\{h^{i}(j(x)) \mid-\infty<i<\infty\right\}$ are disjoint. Let $x_{i}=h^{i}(x)$ and $y_{i}=j\left(x_{i}\right)$. Note that since $j \circ h=h^{-1} j, y_{i}=h^{-i}(j(x))$, so the sets $\left\{x_{i}\right\}$ and $\left\{y_{i}\right\}$ are disjoint. Choose a sequence of intervals $\left\{I_{n}\right\}$ as in [2] and another $\left\{I_{n}^{\prime}\right\}$ such that the length of $I_{n}$ is equal to the length of $I_{n}^{\prime}$. As in [2] we replace $x_{n}$ by $I_{n}$ but then also replace $y_{n}$ by $I_{n}^{\prime}$ thus forming an enlarged circle $\hat{S}$. We define $\hat{j}: \hat{S} \rightarrow \hat{S}$ by $\hat{j}(z)=j(z)$ if $z \notin \cup\left(I_{n} \cup I_{n}^{\prime}\right)$ and in such a way that $\hat{j}$ maps $I_{n}$ isometrically onto $I_{n}^{\prime}$. Now as in [2] define $f: I_{n} \rightarrow I_{n+1}$. The diffeomorphism $k_{2}$ is then defined as follows:

$$
k_{2}(z)= \begin{cases}h(z) & \text { if } z \notin \cup\left(I_{n} \cup I_{n}^{\prime}\right), \\ f(z) & \text { if } z \in I_{n}, \\ j \circ f^{-1} \circ j & \text { if } z \in I_{n}^{\prime} .\end{cases}
$$

Hence $\hat{j} \circ k_{2}(z)=k_{2}^{-1} \circ \hat{j}(z)$ because $j \circ h=h^{-1} \circ j$ and if $z \in I_{n}, \hat{j} \circ k_{2}(z)$ $=\hat{j} \circ f(z)=\hat{j} \circ f \circ \hat{j}^{2}(z)=k_{2}^{-1} \circ \hat{j}(z)$. Q.E.D.

Proof of Theorem. We first construct a foliation with dense leaves. We begin by foliating the two rectangles of Figure 1 by horizontal straight lines. Then the interval $D D^{\prime}$ is attached to $B^{\prime} C$ using the diffeomorphism $g_{1}$ (see Figure 2), i.e. in such a way that if a circle is formed from the interval $B B^{\prime}$ by identifying $B$ and $B^{\prime}$ the diffeomorphism of this circle obtained by sliding along the leaves of the rectangle $B C D^{\prime} D$ is exactly $g_{1}$. The fixed points are $C$ and $B=B^{\prime}$.

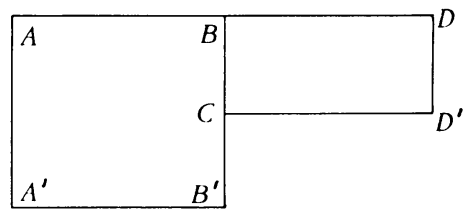

FIGURE 1

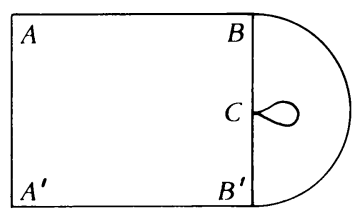

FIGURE 2

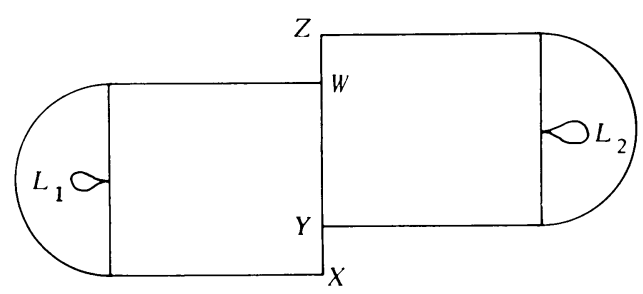

FIGURE 3 


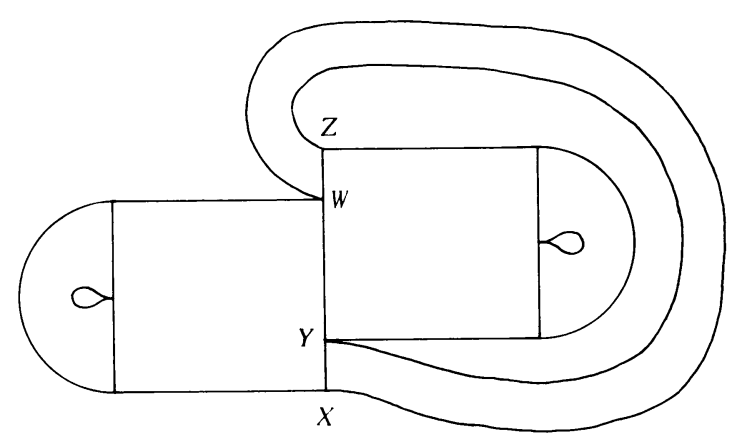

Figure 4

A similar figure using $g_{2}$ is constructed and the two are attached (see Figure 3 ) so that if a circle is formed from the interval $x z$ by identifying the interval $x y$ with the interval $w z$, then the diffeomorphism of this circle obtained by sliding along leaves toward the right (respectively, left) is $g_{1}$ (respectively, $g_{2}$ ). Finally (Figure 4), a foliated strip, is attached joining interval $x y$ to $w z$. If we now consider the diffeomorphism of this circle induced by starting at a point of $x y$ and following its leaf to the right and continuing until the leaf has passed once around each loop $\left(L_{1}\right.$ and $\left.L_{2}\right)$ and returned to $x y$, it is clear that this is simply $g_{1} \circ g_{2}=k_{1}$. Thus we have a foliation with dense leaves except four of its leaves are "branched" manifolds, namely lines with loops on one end. The four loops form the boundary of the region. If this boundary is deleted we obtain a foliation of an open set in the plane which still has each leaf dense.

The foliation with an exceptional minimal set is obtained by exactly the same construction using $g_{3}$ and $g_{4}$ in place of $g_{1}$ and $g_{2}$. Q.E.D.

\section{BIBLIOGRAPHY}

1. G. Hector, Sur un théorème de structure des foliations de codimension un, Thesis, Univ. de Strasbourg, 1972.

2. Z. Nitecki, Differentiable dynamics, M.I.T. Press, Cambridge, Mass., 1971.

3. R. V. Plykin, Sources and sinks of A-diffeomorphisms of surfaces, Mat. Sb. 94 (136) (1974), 243-264 = Math. USSR Sb. 23 (1974), 233-253.

Department of Mathematics, Northwestern University, Evanston, Illinois 60201 